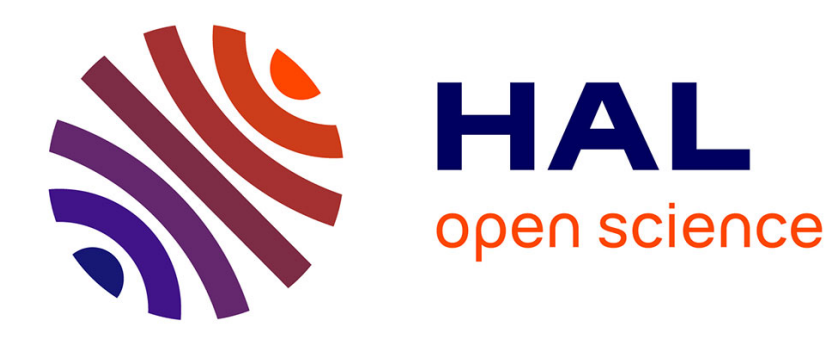

\title{
Rêves et activité transformationnelle de l'adolescent Philippe Givre
}

\section{To cite this version:}

Philippe Givre. Rêves et activité transformationnelle de l'adolescent: Parcours conceptuel de François Ladame. Adolescence, 2009, Psychothérapie IV, 1 (67), pp.177-198. 10.3917/ado.067.0177 . hal01504277

\section{HAL Id: hal-01504277 \\ https://hal.science/hal-01504277}

Submitted on 13 Jun 2017

HAL is a multi-disciplinary open access archive for the deposit and dissemination of scientific research documents, whether they are published or not. The documents may come from teaching and research institutions in France or abroad, or from public or private research centers.
L'archive ouverte pluridisciplinaire HAL, est destinée au dépôt et à la diffusion de documents scientifiques de niveau recherche, publiés ou non, émanant des établissements d'enseignement et de recherche français ou étrangers, des laboratoires publics ou privés. 


\title{
RÊVES ET ACTIVITÉ TRANSFORMATIONNELLE DE L'ADOLESCENT
}

\author{
Parcours conceptuel de François Ladame
}

PHILIPPE GIVRE

Au vu de l'ensemble des travaux et publications de F. Ladame, il serait présomptueux de vouloir analyser de manière exhaustive le parcours conceptuel qui se dégage de son œuvre. Aussi, nous contenterons-nous d'en esquisser certains contours. Ses apports dans le champ de la psychopathologie de l'adolescence - à titre d'exemple, on pense à ses travaux sur la question du suicide à l'adolescence qui font aujourd'hui autorité - sont aujourd'hui plus largement reconnus que dans le champ psychanalytique où la densité et la pertinence de ses positions et réflexions métapsychologiques apparaissent moins clairement identifiées. En effet, F. Ladame ne s'inscrit pas dans la lignée des fervents et prolifiques innovateurs conceptuels - à l'exception notable du concept de «pare-excitations pour le dedans » (1991) qui représente un élément clé de sa conceptualisation des processus adolescents et dont nous aurons à montrer la pertinence - par contre ses écrits attestent d'un véritable ancrage psychanalytique et d'une grande rigueur dans la relecture qu'il propose des concepts métapsychologiques freudiens. On décèle même dans l'ensemble de ses textes, une construction métapsychologique des processus adolescents qui, à notre sens, représente une avancée substantielle sur un certain nombre de points théorico-cliniques. Or, ce

Adolescence, 2009, 27, 1, 177-198. 
sont bien les aspects majeurs de ces modélisations, qui s'efforcent de ne jamais perdre de vue la clinique, dont nous allons essayer d'identifier la portée et la valeur réelle.

\section{MODĖLE DU DÉVELOPPEMENT PSYCHIQUE À L’ADOLESCENCE}

Si J. Lacan énonçait à juste titre qu'une pratique n'a pas besoin d'être éclairée pour opérer, F. Ladame insiste de son côté sur la nécessité pour le clinicien d'être éclairé a minima sur ce qu'il propose à ses patients. Difficile en effet de concevoir qu'un thérapeute puisse «fonctionner efficacement sans modèles : modèle du développement psychique à l'adolescence, modèle de sa pratique ensuite »1. Qu'en est-il donc de ces modèles de développement psychique ?

De façon nettement affirmée, les modèles de référence qu'il propose se situent clairement dans une perspective développementale. S'inscrivant dans la filiation des travaux d'A. Freud ou d'E. Jacobsonses positions s'avèrent assez naturelles, puisqu'elles furent toutes deux les premières à démontrer certaines spécificités de la phase adolescente à partir d'une approche développementale. Par ailleurs, F. Ladame livre des indications intéressantes sur l'influence d'autres auteurs qui ont pu ou ont continué d'exercer une influence significative sur sa pensée. Il en cite notamment trois : deux New-Yorkais, P. Blos et J. Masterson, plus un Londonien, M. Laufer. Aussi, serons-nous naturellement conduit à examiner certains points spécifiques des travaux de ces trois auteurs, afin de préciser ce que sous-tend la revendication d'une approche développementale. S'il ne les ignore pas, F. Ladame, en cela proche d'un certain pragmatisme anglo-saxon, ne s'embarrasse guère des controverses franco-françaises qui alimentent les débats entre perspective structurale et perspective développementale.

Or, on le sait, les critiques à l'égard de cette orientation développementale ont élaboré tout un argumentaire qui vise à sceller un sort rapide et définitif à celle-ci, prétextant tout d'abord que cette orientation reste beaucoup trop tributaire d'un découpage artificiel en tranches de vie, ce qui n'apparaît guère compatible avec une prise en

1. Ladame, 1986, p. 12. 
compte de l'infantile qui doit s'entendre comme élément a-temporel et qui par conséquent ne peut absolument pas se superposer avec la période de l'enfance. Les mêmes auteurs critiques mettront ensuite l'accent sur la propension à octroyer un rôle trop central à l'instance moïque, cette option présentant le risque, selon eux, d'un détournement de l'approche analytique en direction d'une psychologie du Moi, celle-ci se confondant trop souvent avec une visée adaptative.

Moins rétifs à cette orientation, les tenants du processus de subjectivation vont se montrer tout d'abord reconnaissants vis-à-vis de l'introduction de la notion de processus - et notamment de processus de séparation-individuation - mais vont par contre être plus sceptiques quant à la tendance à négliger le rôle de la sphère interne et notamment l'impact de la dimension fantasmatique. Pour F. Ladame, adhérer à cette perspective développementale ne l'empêche pas de mettre au premier plan «l'étonnante activité transformationnelle »² qui s'observe chez l'adolescent, rejoignant du même coup les hypothèses de R. Cahn qui luimême définit l'adolescence comme processus de transformation ou plus exactement un processus de transformation ou de subjectivation qui doit être pensé comme un processus de différenciation davantage que comme un processus de séparation-individuation.

\section{INTERNALISATION DU PARE-EXCITATIONS}

S'il ne renonce pas à l'hypothèse proposée par $\mathrm{P}$. Blos de l'adolescence conçue comme seconde phase de séparation-individuation, F. Ladame y adjoint non seulement des précisions intéressantes, mais en complexifie sensiblement l'élaboration. En effet, si la phase de séparationindividuation de l'enfant dépend étroitement de la qualité de l'environnement, la phase de séparation-individuation de l'adolescent correspond à une réorganisation structurale où l'environnement ne joue qu'un rôle mineur. Aussi, demande-t-il au clinicien d'avoir bien à l'esprit les différences essentielles qui distinguent la première et la seconde phase de séparation-individuation. De ce fait, si «le petit enfant doit créer un espace psychique intérieur et un mode représentationnel à partir de ce

2. Ladame, 1990a, p. 5. 
que lui offre la réalité extérieure; l'adolescent, lui, doit faire un travail de désengagement des objets infantiles internalisés, il doit remanier son monde intérieur, modifier ses introjects (ou pour le moins, les représentations d'objets). Il s'agit alors d'une réorganisation structurale, au cours de laquelle l'environnement - principalement les parents et les groupes de pairs - n'intervient plus qu'à titre d'auxiliaire, si indispensable soit-il $»^{3}$. La réorganisation structurale qui s'opère à l'adolescence va donc être largement dépendante et tributaire des ressources et des fonctionnalités du Moi. Celles-ci vont devoir, comme l'avait souligné P. Blos (1967), maintenir une tension dialectique entre mouvements régressifs et mouvements progrédients, étant entendu qu'à l'adolescence ces mouvements régressifs ne sont pas pathologiques en eux-mêmes et ne peuvent être un critère de diagnostic différentiel.

Or, pour F. Ladame l'adolescence correspond à une période où le sujet doit composer avec un Moi affaibli suite au désengagement du Moi parental. Il en découle que l'une des tâches les plus exigeantes à accomplir par le processus adolescent, tâche qui occupe même une position cardinale, concerne «le relâchement des liens émotionnels infantiles avec les parents $»^{4}$. Il n'y aura donc pas d'adolescence normale sans dépression ou plus précisément sans moments dépressifs, tout au moins tant que la source de sécurité représentée par le Moi parental ne sera pas internalisée. Par conséquent, cette deuxième phase du processus de séparationindividuation devra aboutir à "l'internalisation de la source de sécurité représentée à l'origine par la mère $»^{5}$ et doit être constitutive d'un «pareexcitations pour le dedans ». Cette intégration représentant alors un facteur déterminant pour que le sujet assume les exigences maturatives du processus adolescent, notamment en atténuant la menace du retour massif des objets mauvais refoulés.

On retiendra de tout cela, que si l'enfant a fondamentalement besoin de " sécurité extérieure », notamment dans les cas où il se trouve confronté à des objets spécialement mauvais, l'adolescent quant à lui, aurait prioritairement besoin d'un environnement interne suffisamment

3. Ladame, Snakkers, 1976, p. 547.

4. Ibid., p. 546.

5 Ladame, 1981, p. 15. 
sécure, afin de faire face au chambardement de son monde intérieur. Tel qu'il est pensé par F. Ladame, l'enjeu du processus de séparationindividuation repose fondamentalement sur la possibilité d'internaliser une source de sécurité qui jusque-là était externalisée. La structuration de ce pare-excitations intérieur favorisera ainsi un relâchement du lien aux mauvais objets internalisés, en dénouant l'indéfectible attachement à ces derniers et en modulant l'attirance irrésistible qui émane de ces objets refoulés. Seul ce relâchement sera capable de limiter « cette sorte de possession comme par des esprits diaboliques », ce sentiment de possession étant lié à la hantise du retour de ces mauvais objets internalisés et refoulés.

Loin d'être simplement abstraites ou formelles, les considérations de l'auteur engagent toute la psychopathologie ultérieure qui va dépendre de la conjonction de trois facteurs : " la massivité avec laquelle des objets mauvais ont été installés dans l'inconscient et le degré de mauvaiseté qui les caractérise ; la massivité avec laquelle le Moi s'est identifié avec ces objets mauvais internalisés ; la nature et la force des défenses qui protègent le Moi contre ces objets $\aleph^{6}$. On mesurera donc l'importance de la constitution du pare-excitations du dedans qui va conditionner l'équilibre narcissique du sujet et notamment l'équilibre entre narcissisme de vie et narcissisme de mort : un équilibre qui va permettre ou non de juguler le négativisme de l'adolescent. Eu égard à l'importance du registre du pare-exciations, il est indéniable qu'on se retrouve toujours dans des conditions limites pour l'adolescent. Ce dernier usant fréquemment du recours aux défenses narcissiques pour tenter de parer aux carences ou aux excès de pare-excitations.

Face à l'identification potentiellement massive du Moi aux objets internes - et si le Moi doit être avant tout défini comme Moi corporel - on comprend que la crainte et la hantise d'être possédé par les mauvais objets vont se traduire électivement par des angoisses émanant du Moi-corps et des attaques dirigées à l'encontre de ce même Moi-corps. En d'autres termes, la mauvaiseté du Moi qui fait suite à l'identification massive aux mauvais objets internes ne peut induire par conséquent qu'une déflexion de ce sentiment de mauvaiseté sur le corps qui devient le support ou le

6. Ibid., p. 45. 
réceptacle privilégié pour ces mouvements projectifs. On constate que cette voie était préparée depuis la survenue de la puberté par les modifications survenues à la fois sur un plan physiologique et sur un plan libidinal. De fait, la combinaison des changements corporels et du changement de régime pulsionnel ne peut que favoriser l'émergence d'une dimension « étrangement inquiétante » et faire du corps un objet curieusement auto-séducteur et auto-menaçant. En l'absence de la constitution du pare-excitations interne, le Moi-corps subira un écartèlement tel que la tentation du clivage ou de la dissociation soma/psyché représentera la seule alternative possible, en accentuant de la sorte un mouvement de rejet du corps sexué.

\section{LE REJET INCONSCIENT DU CORPS SEXUÉ}

La référence à la haine du corps sexué convoque immédiatement les apports de M. et E. Laufer qui ont su, les premiers, mettre en évidence le lien entre cassure ou arrêt du développement et rejet inconscient du corps sexué. Pour y parvenir et appréhender les enjeux principaux de l'adolescence, les Laufer nous disent être repartis de l'affirmation toute simple de Freud dans l'Essai sur la puberté : «Les modifications qui surviennent sont destinées à donner à la vie sexuelle infantile sa forme définitive normale. » La puberté doit donc aboutir à l'établissement de l' « organisation sexuelle définitive », dans la mesure où avec «la puberté, commence un processus de développement qui est qualitativement différent de ce qui existait auparavant. La présence du corps sexuellement adulte contraint tout adolescent, que son développement suive un cours normal ou pathologique, à faire des choix inconscients qui, à la fin de l'adolescence, amèneront à une identité sexuelle irréversible (“Irréversible " en l'absence d'intervention thérapeutique bien sûr) »7 . Les orientations prônées par les Laufer s'inscrivent ici très clairement dans le fil des thèses freudiennes développées dans les Trois essais sur la théorie sexuelle qui indiquaient très clairement que l'adolescence devait correspondre à l'avènement du primat du génital, l'acquisition de plaisir se mettant désormais au service de la fonction de reproduction. Initiant

7. Laufer, 1986a, pp. 64-65. 
cette approche développementale, Freud ajoutait que les troubles pathologiques pouvaient être considérés à juste titre comme des inhibitions du développement : «L'arrêt du développement est en luimême la pathologie, en ce sens qu'on observera une interruption de la différenciation du masculin et du féminin (qui constitue la tâche spécifique de l'adolescence) $»^{8}$.

À partir de ce principe, la notion de «breakdown » sera utilisée par les Laufer dans une acception différente de celle proposée par D. W. Winnicott, puisque ce qu'ils nomment « cassure » dans le développement englobe de ce fait un « rejet inconscient du corps sexué », « la haine de son propre corps » et le maintien du sujet dans une posture de victime ou de persécuté. Cette définition du «breakdown » conforte l'idée qu'à l'adolescence la pathologie s'exprime préférentiellement par le biais du corps, qui est la cible privilégiée des attaques destructrices. Par conséquent, « contrairement à l'enfant et à l'adulte, l'adolescent ressent son corps comme la représentation constante de ce qui va le submerger d'émotions et de fantasmes douloureux ou effrayants ${ }^{9}$. D'où la propension du sujet adolescent à être sur ce versant du rejet inconscient afin de mettre à mal le sentiment d'être passif face aux exigences de son propre corps.

Dans le cas de figure où survient le «breakdown», l'impact de cette cassure laisse l'adolescent aux prises avec un sentiment de folie et de destruction de l'épreuve de réalité. Il convient alors de ne pas négliger le risque d'une pathologie future. Certes, les degrés de gravité par rapport à cette rupture n'en restent pas moins variables et gradués. Ils comportent des implications psychiques qui vont d'une simple prédominance du fonctionnement défensif par rapport aux exigences maturatives à une forme d'impasse dans le développement pouvant aboutir à une crise aiguë $\mathrm{du}$ fonctionnement mental, ou plus radicalement encore, pouvant conduire à une fin anticipée du développement, comme dans le cas d'un aménagement pervers.

Quel que soit le cas de figure, pour être en mesure d'appréhender l'ampleur et les conséquences de l'inhibition ou de la cassure du

8. Ibid., p. 78.

9. Laufer M., Laufer E., 1984, p. 39. 
développement chez l'adolescent, la prise en compte du registre fantasmatique s'avérera primordiale. En effet, sans la prise en compte de celui-ci, il sera impossible pour le thérapeute de se faire une idée de la problématique du sujet, puisque le fantasme figure le compromis que le sujet aura pu trouvé au moment de la résolution du complexe d'Edipe. De manière plus précise encore, il conviendra d'accéder au fantasme masturbatoire central du sujet. En l'occurrence, cela suppose de comprendre ce que les Laufer désignent sous les termes de fantasme masturbatoire central.

\section{LE FANTASME MASTURBATOIRE CENTRAL}

Le fantasme masturbatoire central doit être considéré, selon les auteurs, comme un phénomène universel qui ne corrobore pas de façon systématique un versant pathologique. C'est la résolution du complexe d'Edipe qui implique la fixation de ce qu'ils nomment « fantasme masturbatoire central », c'est-à-dire un ensemble fantasmatique dont le contenu correspond aux différentes satisfactions régressives comme aux principales identifications sexuelles du sujet. Si au cours de l'enfance et de la latence son contenu demeure inconscient, il s'exprime néanmoins de façon déguisée à travers les rêveries, les fantasmes qui accompagnent la masturbation, les jeux ou les activités et les relations « comme si ».

L'intégration de ce contenu fantasmatique va s'avérer déterminante d'autant plus qu'après la puberté « un caractère de contrainte s'ajoute à ce fantasme ; il doit être vécu au sein des relations d'objets et dans la vie sexuelle $»^{10}$. Il n'en demeure pas moins que le contenu du fantasme masturbatoire central ${ }^{11}$ ne change pas pendant l'adolescence, même si le fait

10. Ibid., p. 21.

11. Afin de rendre plus explicite la nature du contenu possible du fantasme masturbatoire central, nous citerons d uxexemples proposés par M. et E. Laufer. emier Pexemple : Pour un garçon (le cas Mark) : «Les thèmes être une fil e, que l'on occupe s' de son corps et être humilié étaient étroitement imbriqués dans le fantasme masturbatoire. [...] Il s'identifiait dans ce fantasme à la femme pénétrée par un homme fort et puissant. Le sentiment d'impuissance qui suivait ses masturbations lui procurait de profondes satisfactions ; il avait l'impression que l'on s'occupait alors de lui et qu'il ne faisait temporairement qu'un avec sa mère » (Laufer M., Laufer E., 1984, p. 27).

Deuxième exemple : pour une fille (le cas Jane) : «Il y a un petit homme à l'intérieur d'dle qui l'oblige à se masturber ; après cela, elle a le fantasme honteux qu'un homme l'attrape, l'oblige à lui masser le corps et à sucer son pénis. Elle s'enroule alors sur elle- 
qu'il soit vécu dans le contexte d'un appareil génital achevé signifie que l'organisation défensive est soumise à une tension beaucoup plus grande.

Le fantasme masturbatoire central figure donc le compromis trouvé, au moment de la résolution du complexe d'Edipe entre les mouvements régressifs, les désirs œdipiens et les identifications œedipiennes qui constituent le Surmoi. Mais ce qui intéresse surtout M. Laufer, c'est le destin du fantasme masturbatoire à l'adolescence, soit la solution que trouve l'individu pour intégrer le contenu antérieur et la réalité nouvelle. Pour le dire avec des termes employés cette fois par F. Ladame, il s'agit de savoir comment se réorganise la scène primitive face à la réalité du corps sexuellement mature. Il convient cependant de distinguer une première phase de l'adolescence où persiste un besoin défensif de redevenir passif par rapport au parent œdipien du même sexe et une seconde phase adolescente où l'identification œdipienne au parent du même sexe doit normalement permettre à l'adolescent de surmonter ce mouvement régressif : «Ce n'est qu'à la fin de l'adolescence que l'image du corps est suffisamment stable et intégrée pour que l'adolescent puisse renoncer à utiliser sur un mode défensif la soumission passive au parent œdipien de même sexe comme source de satisfaction $»^{12}$. À l'adolescence, au moment où l'intégration de l'existence de l'appareil génital du sexe opposé est obligatoire, "le refus du corps, soit féminin, soit masculin, signifie qu'on a refusé, de façon irréversible, l'identification œdipienne au parent du même sexe $»^{13}$. Le résultat de ce processus normal étant de parvenir à établir en fin d'adolescence la différence définitive homme/femme, les Laufer fixant ce terme de l'adolescence vers vingt et un an, c'est-à-dire un âge où la qualité de ses relations d'objets est fixée et un âge où les moyens de satisfaction libidinale sont devenus plus spécifiques.

même de manière à ce que sa bouche rejoigne son vagin [...]. Le " petit homme " de son fantasme représentait son père ; mais "le petit homme "était également la figuration de l'agressivite déniée qu'elle exprimait dans l'idée que son vagin contenait du pus ou du poison [...]. Qu'elle ait eu le sentiment que son vagin plutôt que toute autre partie du corps contenait sa puissance destructrice et sa haine est de la plus grande importance pour la compréhension de sa psychopathologie. Sa relation préœdipienne à sa mère et son identification à elle étaient représentées par l'idée de la bouche rejoignant le vagin - c'est-à-dire de la bouche empoisonnant le vagin et vice versa, ainsi que par le fantasme d'un homme l'obligeant à sucer son pénis » (Ibid., p. 51).

12. Laufer M., Laufer E., 1984, p. 43.

13. Ibid., p. 195. 
Pour être en mesure d'appréhender le destin du fantasme masturbatoire central à la puberté et à l'adolescence, le clinicien devra se montrer particulièrement attentif à la question de l'identification du sujet au parent du même sexe. De ce fait, ce sera d'abord ce qui touche à l'Edipe négatif et du même coup à la dimension homosexuelle masochiste qui s'avérera décisif. Le refus de l'identification œdipienne au parent du même sexe participant de la rupture pubertaire, tout en entretenant une relation passive vis-à-vis de ce parent qui elle-même maintient la prédominance de la sexualité infantile chez l'adolescent. Ce refus engendre également un besoin d'agir et de réalisation compulsive, à l'origine de la fréquence du recours à l'acte à l'adolescence. Ces acting out devront alors être compris comme autant de tentatives pour intégrer ce fantasme sur un plan génital.

Disons alors que plus le degré de passivité ou de passivation à l'égard du parent du même sexe est important plus le destin du fantasme masturbatoire central risque d'être engagé sur un versant pathologique. $A$ contrario, plus le versant de l'Edipe positif et avec lui la dimension hétérosexuelle seront présents moins il y aura des motifs d'inquiétude dans la mesure où le fantasme masturbatoire central montrerait par ce biais sa capacité à intégrer la réalité du corps sexué.

\section{«LANGUE, LIMACE, PÉNIS, SAUCISSE ... »}

L'incapacité à intégrer la réalité du corps sexué est bien illustrée par le biais de l'exposition d'un cas clinique que nous propose F. Ladame (1990b). Chez ce patient le désir de féminité envers son analyste, en l'occurrence F. Ladame lui-même, était particulièrement prononcé. Ce désir allait s'exprimer en une première occasion où le patient profitait d'une séance pour apporter des photos d'une soirée costumée pour laquelle il s'était habillé et maquillé en femme, et ceci d'une manière outrancière et hideuse. Cette première initiative était annonciatrice d'un second acting plus parlant encore où cette fois le patient n'hésitera pas à se présenter pour sa séance vêtu de rose des pieds à la tête.

En fait, ce garçon de dix-huit ans qui souffrait de symptômes obsessionnels, se montrait surtout angoissé par des idées parasites comme 
« se regarder regarder qui aurait des conséquences désastreuses pour lui et ses relations ». Ses idées parasites étaient apparues lorsqu'il avait pour la première fois embrassé une fille. Tout s'était alors « confondu, bouche, vagin, anus, avec la panique d'être engouffré corps et bien dans cette bouche-vagin-anus ». Depuis ce moment, qui correspond également à la cassure de développement, il éprouve un besoin compulsif de vérifier par le regard qu'il existe encore. «Dans la bouche de cette fille, il ne savait plus si sa langue était une limace, un pénis, une saucisse... ça tournait si vite. »Chez ce patient, ces éléments se reliaient assez aisément avec d'autres éléments du fantasme masturbatoire central (éléments conscients) qu'il avait pu signifier à d'autres moments du travail thérapeutique. Par exemple, lorsque ce jeune homme décrivait son angoisse d'être absorbé dans un immense vagin, F. Ladame décelait dans la présence de ce fantasme de retour in utero la marque d'une dimension incestueuse.

De façon fondamentale, ce patient était dans l'incapacité de délaisser son « angélisme », c'est-à-dire d'abandonner une image de luimême qui entretenait l'idée d'une perfection du passé infantile. Il était dès lors condamné à rester enfermé dans une soumission au diktat du Surmoi qui n'exige rien de moins que la répudiation du corps du délit, de son corps d'homme puissant. Dans une position particulièrement précaire et inconfortable, son Moi se trouvait écartelé entre les souhaits incestueux qui se réfugiaient au sein de l'Idéal du Moi et les rétorsions du Surmoi à l'encontre des désirs incestueux et parricides.

Sur un plan thérapeutique, on peut considérer que ce patient manifestait un plaisir éventuellement pervers à jouer avec les limites, mais il montrait surtout ce à quoi il se sentait condamné par son thérapeute, convaincu que c'est bien cela que son thérapeute voulait et là qu'ildevait en venir. Si le temps fort de l'analyse, son point organisateur, était celui du scénario masturbatoire central dans lequel le psychanalyste se trouve responsable de la haine de lui-même et de son corps, cela revient à dire que l'analyste en vient à jouer le rôle horrible du parent phallique infantile génitalisé. Or, dans ce cas de figure, le cheminement analytique restera fixé définitivement à ce stade, l'élaboration produite par le patient débouchant sur l'incapacité de désexualiser l'analyste comme objet de 
transfert. De même, il devenait impossible pour le patient d'échapper à une issue non pathologique de la compulsion de répétition. Au-delà du désir de féminité envers son thérapeute, F. Ladame entrevoit un désir de castration, ce désir se superposant à celui de détruire son corps d'homme puissant, histoire de disposer enfin d'un corps qui ne le torturerait plus, enfin pacifié, étant entendu que pour lui la sexualité ne pouvait que rendrefou.

\section{REPRÉSENTATIONS VIVANTES DU COUPLE PARENTAL}

Faut-il dire alors que ce patient était dans l'incapacité d'assumer le fait d'être exclu de la scène primitive et qu'il maintenait encore et toujours la renvendication/conviction d'être « dans » le couple, d'être coparticipant du coït parental au point de s'y perdre et d'y être englouti ?

Le destin du fantasme masturbatoire central est lié, on le sait, à la possibilité pour le sujet adolescent de tolérer l'exclusion de la scène primitive. Mais, et c'est là une précision déterminante qu'apporte F. Ladame, c'est à la condition de maintenir vivante la représentation du couple parental après que le sujet s'en soit exclu. "Quant à l'adolescence névrotico-normale, je la définirais comme la réussite de l'assomption d'une position de sujet désirant/désiré, ce qui implique la constitution d'une structure de désir, passant par la résolution (et non la simple mise en veilleuse) du conflit odipien, autrement dit la possibilité de maintenir vivante la représentation de la relation du couple parental après s'en être exclu. Pour moi, c'est là la véritable innovation, pourquoi ne pas dire même révolution de l'adolescence ! ${ }^{14}$. À ce titre, on peut dire que «tant que le clivage n'opère pas de façon trop radicale, le conflit peut être maintenu vivant $\gg 15$.

Effectivement, dans ce moment où se réorganise la scène primitive face à la réalité du corps sexuellement mature, le recours à la fonction onirique va se révéler déterminant. F. Ladame n'hésitera pas à affirmer que l'achèvement et l'intégration du corps sexué suppose un fonctionnement adéquat du travail du rêve. Or, si le rêve est utile avant la puberté, il devient indispensable quand la maturité est achevée : «Si le

14. Ladame, 1987, p. 104.

15. Ibid., 108. 
rêve remplit sa fonction, il constitue la " voie royale " où les désirs cedipiens se pérennisent en toute méconnaissance et s'assouvissent en toute impunité »16. Par conséquent : «L'achèvement du développement du corps, nécessite un fonctionnement adéquat du travail du rêve (qui est l'essence $d u$ rêve) ${ }^{17}$. Un tel présupposé théorique revient à envisager une fonction originelle du rêve qui ne serait pas de l'ordre de la satisfaction d'un désir : "Mais quelle serait la fonction originelle du rêve si ce n'est pas la satisfaction d'un désir? Opérer une liaison, faire office de pareexcitations pour le dedans. Ceci étant garanti, le couple régulateur plaisir-déplaisir peut prendre tous ses droits. L'activité de liaison est une condition préliminaire pour que le principe de plaisir entre en jeu et assure sa dominance $\gg^{18}$.

Lorsque le travail du rêve ne parvient pas à susciter ce processus de liaison/déliaison/reliaison, le rêve est moins l'aboutissement d'une élaboration que le reflet d'une fonction d'évacuation et d'un déficit de l'appareil à penser les pensées. F. Ladame se ressaisit ici des travaux de L. Grinberg et de son groupe de recherche qui «ont étendu au rêve le concept bionien d'appareil à penser les pensées et ont forgé l'idée d'un " appareil à rêver les rêves ", dont la fonction serait double : d'abord rêver le rêve pendant le sommeil, ensuite penser à ce rêve à l'état de veille, c'est-à-dire se le remémorer plutôt que le répéter en acte »19. Aussi, les rêves qui remplissent le mieux leur fonction et qui témoignent de la présence efficiente d'un appareil à rêver les rêves, sont ceux, en définitive, dont on ne sait plus rien après le réveil. À l'inverse, les rêves traumatiques, qui restent sous l'emprise de la compulsion de répétition signent l'échec total de la fonction onirique, représentant par là même de véritables « antirêves ». Le défaut d'un appareil à rêver les rêves dont ils témoignent se traduira prioritairement par des acting, étant donné que l'acting out sera pensé comme l'équivalent d'un « rêve qui n'a pas pu être rêvé »20.

En outre, il convient de ne pas confondre acting et « expérience »: «La distinction entre " expérience " et " acting " ne doit pas non plus

16. Ladame, 1991, p. 1537.

17. Ibid., p. 1502.

18. Ibid., p. 1533.

19. Ibid., p. 1519.

20. Ibid., p. 1524. 
être perdue de vue. L'expérimentation est au service de l'intégration, notamment de la nouvelle représentation du corps. L'agressivité est alors liée dans l'activité, qui devient relation à un corps actif. L'acting signale - au dehors - la non-résolution du conflit lié à l'intégration. L'investissement forcené du monde extérieur joue fréquemment un rôle anti-intégrateur $»^{21}$. Là où l'acting vient traduire un dysfonctionnement du préconscient, l'activité de rêverie reflétera, de son côté, la souplesse du jeu entre refoulement et retour du refoulé. Or, c'est bien cette fonctionnalité du préconscient et cette souplesse qui sont requises pour maintenir vivantes les représentations du couple parental.

Paradoxalement, des instants de dysfonctionnalité de cette même instance, se traduisant par des sentiments " d'inquiétante étrangeté », doivent aussi pouvoir être tolérés par le sujet adolescent. Un processus d'adolescence sera d'autant moins problématique que pourront se conjuguer durant cette phase fonctionnalité et moments de dysfonctionnalité. De fait, les moments qui signent un certain vacillement de l'épreuve de réalité, se manifestant par des impressions de « déjà vu » ou de "déjà vécu » viennent signifier «que les voux incestueux et parricides ne sont pas encore confinés à l'Inconscient, qu'ils font temporairement intrusion dans la Conscience sans être maquillés par le Préconscient. Ils traduisent un travail en cours de refonctionnalisation du Préconscient ${ }^{22}$. Il appartiendra donc à la fonctionnalité du Préconscient d'assurer à la fois la possibilité de tolérer la présence masquée des scènes pubertaires - c'est-à-dire d'assumer une certaine levée du refoulement et une certaine transparence psychique - tout en étant en mesure de rerefouler ces scènes ou fantasmes incestueux et parricides. Lorsque ce compromis ne parviendra pas à être aménagé, l'impossibilité de rerefouler les fantasmes pubertaires équivaudra immédiatement à un risque pathologique : "Ce qui fait la caractéristique de la pathologie, c'est l'impossibilité de re-refouler les scènes pubertaires, par essence crues et cruelles, et, par là même, désorganisantes. Est en cause ici la fonctionnalitédu Préconscient. Selon sa qualité pourront survenir dans la

21. Ladame, 1985, p. 157.

22. Ladame, Perret-Capitovic, 1997, p 242. 
suite du processus des moments d'affaissement de la topique (moments de raptus par exemple, ou moments de cassure) qui s'apparentent à la catégorie du traumatique $»^{23}$.

Dans ces moments « d'affaissement de la topique » et à défaut de la restauration des mécanismes propres au refoulement, on peut s'interroger sur la nature et les modalités défensives qui resteront à la disposition du sujet. Ainsi, en plaçant au cœur de sa réflexion la notion de « représentation vivante du couple parental » F. Ladame dialectise implicitement cette dernière avec les notions de représentation inanimée, fétichisée, voire de représentation spectrale de l'autre ou du couple parental. La logique implicite à cette relation dialectique reposerait par conséquent sur le postulat suivant : plus l'inquiétant, l'inanimé, le fantomatique ou le spectral seraient présents dans la vie psychique du sujet, plus ils attesteraient d'un déficit de la fonctionnalité du Préconscient pouvant compromettre la possibilité de maintenir vivantes et animées les figures internalisées du sujet.

En d'autres termes, la singularité de ce qui se jouerait à l'adolescence concernerait bien le retour sur la scène psychique d'un Edipe fantomatique. Ceci se matérialiserait par un CEdipe plus «blanchi » que refoulé, c'est-à-dire un Edipe qui mobiliserait moins le contreinvestissement que la propension à faire appel au désinvestissement qui ouvre lui-même la voie au narcissisme de mort. On verra donc apparaître ces nouvelles modalités œdipiennes lorsque la sélectivité du refoulement n'aura pas permis aux désirs incestueux et parricides d'être négativés, sans que toute la sexualité succombe au passage. L'enjeu paradoxal serait que le sujet puisse être en mesure de négativer l'Edipe (au sens de sa destruction puisque les éléments œdipiens dans ce corps nouveau sont intolérables) et en même temps de réussir à redonner quelques couleurs à ces mêmes représentations fantasmatiques afin de les maintenir vivantes.

CHANGEMENT DE PRINCIPE DE RÉALITÉ

La capacité de maintenir vivantes ces représentations suppose un cheminement exigeant, lequel engage à la fois la fonctionnalité du

23. Ibid., p. 240. 
Préconscient et la mise en jeu du travail du négatif. C'est grâce à l'action conjointe de ces processus que le changement de principe de réalité deviendra tolérable, au même titre que le changement de régime pulsionnel, lequel caractérise la période adolescente. Si l'idée d'un changement de régime pulsionnel devient plus explicite, grâce aux propositions des Laufer, il nous reste à expliciter l'hypothèse d'une modification sensible du principe de réalité. La puberté constitue en effet un point de bascule entre principe de réalité de l'enfance et principe de réalité de l'adolescence. Autant le principe de réalité de l'enfant repose fondamentalement sur l'impuissance du corps - celle-ci étant contrebalancée par la toute-puissance de la pensée (protectrice du narcissisme blessé de l'enfant) -, autant le principe de réalité de l'adolescent repose quant à lui sur la puissance du corps (au sens orgasmique du terme) et engage la dissolution de la toute-puissance de la pensée, ainsi que la reconnaissance de la finitude, de la mort, de la différence des sexes et de la différence des générations. La mégalomanie infantile créerait par conséquent ce monstre auquel les adolescents ont le plus de difficultés à couper la tête.

Ce qui peut surprendre toutefois, c'est la vision assez péjorative que F. Ladame développe à propos de l'enfance et des pouvoirs de l'autoérotisme. En l'occurrence, il affirme que la vraie quête objectale, celle qui permet de sortir de l'auto-érotisme et de renoncer à la solution de facilité des plaisirs dits solitaires, est celle qui est différée jusqu'à la puberté. Il insiste par ailleurs sur l'idée que l'enfance occasionne davantage de frustrations et de déplaisirs que d'expériences de plaisir. L'enfance serait donc pour lui avant tout marquée du sceau de la « révolte » face à la néotonie et à l'impuissance infantile, au point que celle-ci deviendrait le vecteur de tout le fonctionnement psychique. F. Ladame se dit d'ailleurs « frappé par la difficulté de beaucoup de collègues à admettre l'idée, défendue par $S$. Freud, que les expériences infantiles ont surtout provoqué $d u$ déplaisir ${ }^{24}$. Dès lors, il convient de ne pas se laisser abuser par les idéalisations défensives du passé infantile en se rappelant que « la première floraison de la vie sexuelle infantile » (Freud, 1920g) a laissé le

24. Ladame, 1998, p. 81. 
goût amer de l'échec. "La sexualité infantile est marquée du sceau de l'infériorité, de l'impuissance et du tragique (Freud, 1920g) $\gg^{25}$. Au regard de ce constat, l'élation adolescente pourrait être comprise, au moins partiellement, comme la possibilité entrevue pour le sujet de s'affranchir enfin de cette cruelle triade infantile.

\section{CONCLUSION}

Afin de caractériser l'un des enjeux majeurs de l'adolescence, F. Ladame a pu s'appuyer sur certaines propositions de C. F. Rycroft qui énonçait que la tâche première du processus adolescent était de réduire "l'antagonisme entre les facultés imaginatives et les capacités d'adaptation ${ }^{26}$. Ce gain psychique devant lui-même se traduire par une plus grande autonomie vis-à-vis du monde extérieur ainsi qu'une meilleure différenciation entre soi et autrui. Pour parvenir à ces fins, il conviendra que l'adolescent puisse rester en contact avec «son étonnante activité transformationnelle $»^{27}$.

Si on peut reconnaitre dans ce type de formulation la marque d'une perspective développementale, clairement revendiquée par F. Ladame (au sens où Freud assignait une ligne développementale à la libido, ligne qui devait conduire au primat du génital), nous avons vu que cette perspective n'occultait en aucune façon l'importance du registre fantasmatique ou de la réalité interne. À l'inverse, c'est même la prégnance du paradigme du rêve dans son œuvre que nous aurons cherché à mettre en évidence. Plus précisément, il s'est agi de pointer l'importance du travail du rêve qui semble pouvoir être assimilé au travail du Préconscient. Cette quasiéquivalence laisse mieux percevoir l'importance que F. Ladame octroie, dans un registre topique, à la fonctionnalité du Préconscient. En effet, il revient au travail du rêve et/ou à la fonctionnalité du Préconscient de garantir un environnement interne « suffisamment bon » et sécurisant afin que le sujet bénéficie d'une certaine tranquillité d'esprit. Encore faut-il que l'internalisation du «pare-excitations pour le dedans » ait été rendue

25. Ladame, Perret-Capitovic, 1997, p. 236.

26. Ladame, 1980, p. 572.

27. Ladame, 1990, p. 5. 
possible lors de la seconde phase de séparation-individuation, puisqu'elle seule sera capable de moduler l'écartèlement que le Moi doit subir lors de la phase adolescente. En l'absence de cette structuration préalable ou de cette organisation primitive d'un Moi, agent du processus transformationnel, il ne subsistera alors pour le sujet que la possibilité de recourir à des mécanismes défensifs qui s'avéreront extrêmement coûteux. Ces derniers engageront tantôt la nécessité de faire tourner à fond « la machine à refouler » (comme chez les jeunes suicidants), tantôt le recours intensif à l'identification projective laquelle contrarie toute possibilité d'identification introjective et, avec elle, bloque tout processus d'internalisation : le clivage du Moi constituant alors la seule issue pour se défendre des menaces d'annihilation qui le submergent.

Ainsi, dans l'œuvre de F. Ladame on voit s'entremêler très nettement les rôles et fonctionnalités du Préconscient, du travail du rêve, $\mathrm{du}$ « pare-excitations pour le dedans ». Toutes ces intrications réhabilitant du même coup le rôle du Moi au sein du processus adolescent. Il est en effet dévolu à l'instance moïque de trouver des compromis capables d'intégrer à la fois le nouveau principe de réalité lié à l'émergence de la génitalité et de tolérer la présence maintenue vivante de fantasmes incestueux et parricides : "L'inceste est au cour de l'adolescence $»^{28}$. $\mathrm{C}$ 'est en ces termes que F. Ladame avec Freud nous rappellent que les désirs dans l'Inconscient sont pour ainsi dire immortels : «La question de l'inceste et du parricide n'en exige pas moins une solution, car ces désirs ne peuvent être "perdus " (Freud, 1900a). Cette solution fait nécessairement appel au refoulement et au Préconscient, c'est-à-dire à un compromis, à un artifice : au regard de son Surmoi, le sujet renonce à ses désirs oedipiens tout en laissant ceux-ci subsister dans l'Inconscient $\gg^{29}$.

28. Ladame, 1991, p. 1499.

29. Ladame, Perret-Capitovic, 1997, pp. 236-237.

Freud soulignait déjà dans les Trois essais l'importance de l'affranchissement de liutorité parentale. Or, ce détachement passe par la modification du contenu du Surmoi. Le Surmoi va donc jouer un rôle décisif dans le succès ou l'échec de la tentative de lảdolescent d'assurer son organisation sexuelle définitive. La « désorganisation » des relations entre le Moi et le Surmoi qui se produit à l'adolescence - les Laufer parlent de détachement du Moi à l'égard du Surmoi - serait responsable d'une augmentation du danger pulsionnel. «Pendant la puberté, la relation du Surmoi au Moi est rompue, et il faut trouver un moyen de rétablir cette relation pour qu'elle inclue une identité sexuelle acceptable pour le Surmoi » (Laufer M., Laufer E., 1984, p. 96). 
Chez nos deux auteurs, la dimension inconsciente loin d'être reléguée au second plan, s'avère omniprésente au point même de constituer le catalyseur de toute l'économie psychique. Cependant, elle reste tributaire de cet « échangeur » privilégié qu'est le Préconscient. Si le Moi agent ne peut, en effet, être dissocié de l'emprise exercée par le "Cogito orgasmique » au moment de la puberté, son action pour se libérer de cette emprise du Ça ne pourra s'exercer pleinement qu'à partir de ce postefrontière que représente le Préconscient.

Sous l'influence du cogito orgasmique, le développement de l'adolescence devra aboutir à une transformation de l'autoreprésentation du sujet, celle-ci intégrant la double notion de corps génital et de complémentarité des sexes. En cela, F. Ladame est proche ici des thèses K. R. Eissler et des Laufer, qui font prévaloir le paradigme de l'orgasme sur celui de l'auto-érotisme. Or, pour F. Ladame il ne semble pas excessif ni même réducteur d'indiquer que l'auto-érotisme ainsi que l'omnipotence de la pensée infantile sont d'abord là pour permettre une « survie narcissique » face au camouflet de l'impuissance infantile.

L'orgasme devient donc le prototype de tout acte, de toute action. Toutefois, il convient de penser le recours à l'acte - notamment sous la forme d'actes d'auto-sabotage - comme procédé anti-orgasmique, « en opposition à l'action dont le prototype serait l'orgasme. N'oublions pas pour autant que le moment de la cassure et l'entrée en jeu de la compulsion de répétition coüncide avec la "réalisation " d'un scénario incestueux et parricide $\gg^{30}$. La composante incestueuse demeure toujours présente, y compris dans les formes les plus graves de cassures du développement. De la sorte, $F$. Ladame se rallie très clairement aux thèses des Laufer, lesquels infèrent que même lorsqu'ils parlent de cassure ou de «breakdown », l'adolescent présente une défense contre des fantasmes incestueux, et cela quel que soit le tableau clinique. M. Laufer affirme même que « cela est vrai en toutes circonstances »31! On peut apporter une nuance en précisant qu'une différenciation existe entre fixation préœdipienne et régression à une phase préœdipienne du développement

30. Ladame, 1991, p. 1537.

31. Laufer, 1986b, p. 108. 
consécutive à l'impossibilité d'assumer l'intégration des attributs de la puissance sexuelle. Mais, s'il existe un point sur lequel F. Ladame ne variera pas tout au long de son œuvre, ce point concernera électivement sa compréhension du contenu fantasmatique dont la compulsion à répéter est le témoin : «la persistance du vou incestueux et parricide à l'état brut (non lié) $»^{32}$.

Loin de revendiquer une quelconque exhaustivité dans l'approche du parcours conceptuel que propose F. Ladame, nous avons simplement souhaité ouvrir quelques pistes de réflexion et apporter un éclairage nouveau sur des hypothèses innovantes et marquantes de l'auteur. Entre autres, la fonction paradigmatique du rêve et sa fonction pleinement assumée, comme signe d'une assomption du pubertaire, nous a paru la plus opérante et la plus révélatrice de la singularité de sa pensée : chez lui, le travail du rêve est placé au cœur du travail de subjectivation. Dans la lignée de ses hypothèses les plus probantes, celle qui envisage la puberté comme instigatrice d'un changement de principe de réalité - étant entendu que «l'épreuve de la réalité n'est autre que la vérité du corps »33-nous semble également particulièrement pertinente. La perte de l'illusion de perfection, comme de l'illusion de bisexualité, constitue donc un préalable à l'investissement possible d'un corps d'homme ou de femme, tel que la puberté l'impose sans choix possible.

En dernier lieu et dans une perspective plus directement liée à la clinique, nous voudrions souligner l'importance que revêt pour l'auteur la réelle prise en compte de la souffrance psychique d'autrui. En témoigne, par exemple, ses propos introductifs à l'ouvrage Les tentatives de suicide des adolescents (1981) où, il mentionne à quel point la souffrance humaine atteint chez ces patients une expression dramatique hors du commun, proche dans son intensité des souffrances psychotiques. En outre, F. Ladame n'hésite pas non plus à confier au lecteur à quel point leur souffrance a pu personnellement le pénétrer, et parfois même le torturer presque autant qu'eux. Or, il n'est pas très fréquent de rencontrer un auteur qui puisse s'avancer aussi courageusement sur le plan de ses

32. Ladame, 1991, p. 1524.

33. Ladame, 1985, p. 156. 
éprouvés contre-transférentiels. De même, il n'est pas courant que soit réaffirmé de façon aussi tranchée que tout thérapeute a beaucoup à apprendre sur le fonctionnement psychique de ses patients, même en situation d'échec thérapeutique. F. Ladame n'hésitant guère à utiliser comme objet de recherche ses propres difficultés et échecs.

\section{BIBLIOGRAPHIE}

BLOS P. (1967). Adolescence et second processus d'individuation. In : M. PerretCatipovic, F. Ladame (Éds.), Adolescence et psychanalyse : une histoire. Lausanne/Paris : Delachaux \& Niestlé, 1997, pp. 113-150.

LADAME F. (1980). L'adolescent en psychanalyse : résistances intérieures et résistances extérieures. Rev. Fr. Psychanal., 44 : 571-579.

LADAME F. (1981). Les tentatives de suicide des adolescents. Fribourg : Masson.

LADAME F. (1985). Rupture ou discontinuité ? Adolescence, $3:$ 155-158.

LADAME F. (1986). Quels adolescents soigner et comment? In : F. Ladame, P. Jeammet (Éds.), La psychiatrie de l'adolescence aujourd'hui. Paris : PUF, pp. 9-16.

LADAME F. (1987). Les états psychotiques à l'adolescence. Adolescence, 5 : 95-108. LADAME F. (1990a). Traitement des adolescents psychotiques : les difficultés et les pièges

In :F. Ladame, Ph. Gutton, M. Kalogerakis (Éds.), Psychoses et adolescence. Paris : Masson, pp. 1-8.

LADAME F. (1990b). Adolescence et compulsion de répétition. Bulletin de la Société

Suisse de Psychanalyse, 30 : 17-23.

LADAME F. (1991). L'adolescence, entre rêve et action. Rev. Fr. Psychanal., 55 : 1491-1542. LADAME F. (1998). Qu'appelle-t-on amour de transfert ? Adolescence, 16 : 75-83.

LADAME F., PERRET-CAPITOVIC M. (1997). Le normal et le pathologique à l'adolescence.

In : M. Perret-Catipovic et F. Ladame (Éds.), Adolescence et psychanalyse : une histoire. Lausanne/Paris : Delachaux \& Niestlé, pp. 229-245.

LADAME F., SNAKKERS J. (1976). Réflexions sur la vie dans les foyers de semi-liberté et sur la place de l'adolescent dans la société. Psychiatr. Enfant, 19: 543-576.

LAUFER M. (1986a). Psychopathologie de l'adolescent et objectifs thérapeutiques. In :

F. Ladame, P. Jeammet (Éds.), La psychiatrie de l'adolescence aujourd'hui. Paris :

PUF, pp. 61-86.

LAUFER M. (1986b). Première table ronde. In : F. Ladame, P. Jeammet (Éds.), La psychiatrie de l'adolescence aujourd'hui. Paris : PUF, pp. 87-111.

LAUFER M., LAUFER E. (1984). Adolescence et rupture du développement. Paris : PUF, 1989. 
Philippe Givre

Équipe de Recherches sur l'Adolescence

Université Paris VII - Denis Diderot

U.F.R. Sciences Humaines Cliniques

26, rue de Paradis

75010 Paris, France

Hôpital Saint-Vincent-de-Paul

Service de psychiatrie

Bd. de Belfort

59020 Lille, France

philippegivre@free.fr 